\title{
Spatial matching and nonmatching in male and female Wistar rats: Effects of delay-interval duration
}

\author{
FRANS VAN HAAREN and ANNEMIEKE VAN HEST \\ Netherlands Institute for Brain Research, Amsterdam, The Netherlands
}

\begin{abstract}
Male and female Wistar rats were trained in a delayed matching-to-position procedure in which one of the two levers (sample) was presented. Pressing this lever resulted in its retraction and began a delay interval of random variable duration, which terminated with the occurrence of the first nose poke in the pellet retrieval unit after the delay interval had expired. Both levers were then inserted into the chamber, and food became available when the subject pressed the lever that had previously been pressed (matching response). When the subject failed to make a matching response, time out $(5 \mathrm{sec})$ was presented. In the next experimental condition, nonmatching was reinforced. Males and females required an equal number of trials to attain $80 \%$ accuracy during three consecutive sessions under matching and nonmatching conditions. Response accuracy decreased as the delay interval increased, during both conditions. Differences between the sexes were not observed, suggesting that memory functions in male and female rats may only differ when other behavioral differences between the sexes are allowed to interfere with the assessment of memory functioning.
\end{abstract}

Memory of recent events has been investigated in numerous experimental procedures, whether positively or aversively motivated. In such procedures, behavior at time $X$ is expected to be under discriminative control of stimuli that were present at time $X-t$, but not at the time of response execution. The stimuli controlling the behavior can be visual, auditory, or tactile, or even a record of the subject's own (spatially defined) behavior on a previous trial (van de Poll, de Bruin, van Dis, \& van Oyen, 1978; van Haaren, de Bruin, Heinsbroek, \& van de Poll, 1985; van Hest, van Haaren, \& van de Poll, 1988a; van Hest, van Kempen, van Haaren, \& van de Poll, 1988b).

When male and female rats are exposed to complex mazes (Lashley III, Hebb-Williams), males appear to behave more efficiently than females (Barrett \& Ray, 1970; Davenport, Hagquist, \& Rankin, 1970; Dawson, Cheung, \& Lau, 1975; Krasnoff \& Weston, 1976). It has been hypothesized, however, that females make more errors than males in these experimental procedures, because of their higher levels of baseline locomotor activity (Beatty, 1979; van Haaren, Wouters, \& van de Poll, 1987). Conversely, females display better retention than males when aversively motivated. In several experiments, females, but not males, have remembered the location of aversive stimulation in passive avoidance choice procedures (Heinsbroek, Feenstra, Boon, van Haaren, \& van de Poll,

\footnotetext{
This research was conducted while Annemieke van Hest was supported by a grant from the Dutch Organization for the Advancement of Basic Research (ZWO 560-258-024) awarded by Frans van Haaren, Correspondence may be addressed to A. van Hest, Behavioral Neuroendocrinology Unit, Netherlands Institute for Brain Research, Meibergdreef 33, $1105 \mathrm{AZ}$ Amsterdam, The Netherlands.
}

in press; van Haaren \& van de Poll, 1984a, 1984b, 1986). In other experiments, however, males have shown more behavioral inhibition following shock presentation than females have (Heinsbroek, van Haaren, \& van de Poll, 1988). It may thus be the case that behavioral differences between the sexes in procedures involving aversive motivation may be determined by the nonspecific effects of shock presentation per se, and not by other experimental parameters. These procedures are thus not ideally suited for investigating memory processes in male and female rats.

To prevent sex differences in locomotor activity and reactions to aversive stimulation from interfering with the assessment of sex differences in memory functioning, operant delayed spatial response alternation procedures have been employed to study memory in male and female rats (van de Poll et al., 1978; van Hest et al., 1988a, 1988b). Although sex differences in steady-state response accuracy were not observed in these experiments, males did acquire efficient alternation performance faster than females did. Furthermore, it was shown that the presentation of stimuli during the delay interval of a spatial delayed alternation procedure disrupted the response accuracy of males more than that of females. It has been suggested that these behavioral differences between the sexes may have been a function of the fact that males were more likely than females to acquire response chains. Delayed spatial operant response alternation procedures allow subjects to develop consistent response chains because the spatial location of responses leading to food presentation is accurately predictable once a response has been reinforced. Previous experiments have suggested that males are more likely than females to engage in lever- 
directed activities in an operant environment, whereas females are more likely to engage in behavior other than lever-directed activities (van Hest, van Haaren, \& van de Poll, 1987a, 1987b). Higher levels of lever-directed activity might facilitate the development of consistent response chains, which in turn would lead to a facilitated acquisition of operant delayed spatial alternation performance.

The present experiment was designed to prevent the development of response chains by varying the location of sample presentations within each experimental session. Male and female Wistar rats were thus exposed to an operant delayed spatial matching procedure, in which presentation of the sample stimulus-one of the levers-was terminated when subjects pressed it. Food was presented when subjects pressed the same (matching) lever, or, in the next phase of the experiment, when they pressed the opposite (nonmatching) lever during presentation of both levers as comparison stimuli. This occurred after the expiration of a variable $(0,5,10$, or $20 \mathrm{sec})$ delay interval. Training on an operant delayed spatial nonmatching procedure followed. In both conditions, subjects were required to terminate the delay interval by engaging in behavior other than lever pressing (nose pokes). These manipulations were introduced to ensure that both males and females engaged in similar activities during the delay interval.

\section{METHOD}

\section{Subjects}

Seven male and 7 female Wistar rats, obtained from Animal House, TNO (Zeist, The Netherlands) participated in the experiment. They were housed in group cages ( 3 or 4 same-sex subjects to a cage) under a reversed light:dark cycle (lights on 6:30 p.m.-6:30 a.m.). The subjects were food-deprived and fed daily to maintain body weights at approximately $85 \%$ corrected for growth throughout the experiment (Hurwitz \& Davis, 1983). Water was always available in the homecages. The subjects had previously participated in an experiment designed to investigate the development of cooperative behavior (van Hest \& van Haaren, 1988). They were 22 weeks old at the start of the experiment.

\footnotetext{
Apparatus

The experiment took place in eight locally constructed rat chambers ( $34 \mathrm{~cm}$ wide, $33 \mathrm{~cm}$ long, and $37 \mathrm{~cm}$ high). The sidewalls and intelligence panel were made of black Perspex. The front door of the chamber was made of translucent Plexiglas. The floor consisted of 26 grids, spaced $1.3 \mathrm{~cm}$ apart. Two retractable rodent levers ( $2.5 \mathrm{~cm}$ long, $2.8 \mathrm{~cm}$ wide, and $0.75 \mathrm{~cm}$ thick, when extended) were located symmetrically, beside the pellet-retrieval unit. The levers required a force in excess of $.20 \mathrm{~N}$ to be operated: each leverpress operated a Sonalert for $0.10 \mathrm{sec}$. A stimulus light (green on the left and red on the right side of the intelligence panel) was located $9 \mathrm{~cm}$ directly above each lever. The pellet-retrieval unit, which was located between the two levers, could be illuminated by a white light, while an infrared light detector was used to record tray entries (nose pokes). A houselight was mounted in the middle of the intelligence panel, $3 \mathrm{~cm}$ from the ceiling of the chamber. All experimental chambers were enclosed in a sound-attenuated, ventilated cabinet; the front door of this cabinet was also made of translucent Plexiglas. The chambers were connected to a DEC PDP 11-73 microcomputer, located in an adjacent room. Experimental con-
}

tingencies and data acquisition procedures were programmed using SKED- 11 (Snapper \& Inglis, 1985), obtained from State Systems, Inc. (Kalamazoo, MI).

\section{Procedure}

Preliminary training. Leverpress training was not necessary, since all the subjects had previously participated in another experiment. In that experiment, however, leverpress training had been restricted to either the left or the right lever, for individual subjects. The subjects thus received four sessions of additional training in which the left and right levers were randomly presented, and in which every second (fixed-ratio [FR] 2, two sessions) or fifth (FR 5, two sessions) leverpress was immediately followed by the presentation of a 45-mg BioServe food pellet. All subjects pressed both levers reliably upon their presentation at the completion of this preliminary experimental condition.

Delayed matching-to-position. The houselight was illuminated at the start of each session. A trial started with the initiation of a fixed 5.0-sec intertrial interval (ITI). Once the ITI had expired, either the left or the right lever was randomly inserted into the experimental chamber, while the associated stimulus light was also illuminated. A response on this lever (sample response) resulted in the retraction of the lever from the chamber, extinguished the stimulus light, and started the delay. A sample response also illuminated the light in the food tray. The light in the food tray was extinguished, both levers were reinserted into the chamber, and the stimulus lights above the levers were illuminated, immediately, upon the first nose poke after the expiration of the delay. A press on the lever on which the delay had been initiated (matching response) was immediately followed by the retraction of the levers, the presentation of a 45 mg BioServe food pellet, and the start of the next ITI. A response on the other lever (incorrect response) produced a time-out (TO) for $5 \mathrm{sec}$. During TO the levers were retracted from the chamber, and the stimulus lights and the houselight were extinguished. After the TO and the ITI, the trial that the subject had just completed incorrectly was repeated. This sequence continued until a correct response occurred.

All subjects were exposed to four different delay intervals within each experimental session: $0,5,10$, and $20 \mathrm{sec}$. These delay intervals were randomly presented, such that the same interval was never presented more than three times in a row. The subjects were exposed to this experimental condition for 30 sessions; the percentage of correct responses during sessions 25-30 did not differ from that observed during sessions $20-25$, for either males or females.

Delayed nonmatching-to-position. Subjects were exposed to a delayed nonmatching-to-position procedure in the next 30 sessions. This procedure was basically identical to the one used in the previous experimental condition, except that correct responses were recorded when subjects pressed the lever not pressed during the sample part of the trial (nonmatching). An incorrect matching response was recorded when the subjects pressed the lever also pressed during the sample part of the trial.

All sessions ended after 30 min or after 60 reinforcers had been presented, whichever came first. Sessions were run 5 days a week (Monday through Friday), during the subjects' dark hours. Figure 1 presents a schematic overview of the experimental procedure.

\section{RESULTS}

Figure 2 shows the percentage correct matching and nonmatching responses (correct responses/correct + incorrect responses) during the different delay intervals and experimental conditions. The data presented are based on the results of 7 female and 6 male rats, because 1 of the males showed a consistent position bias during the first experimental condition. The percentage of correct 


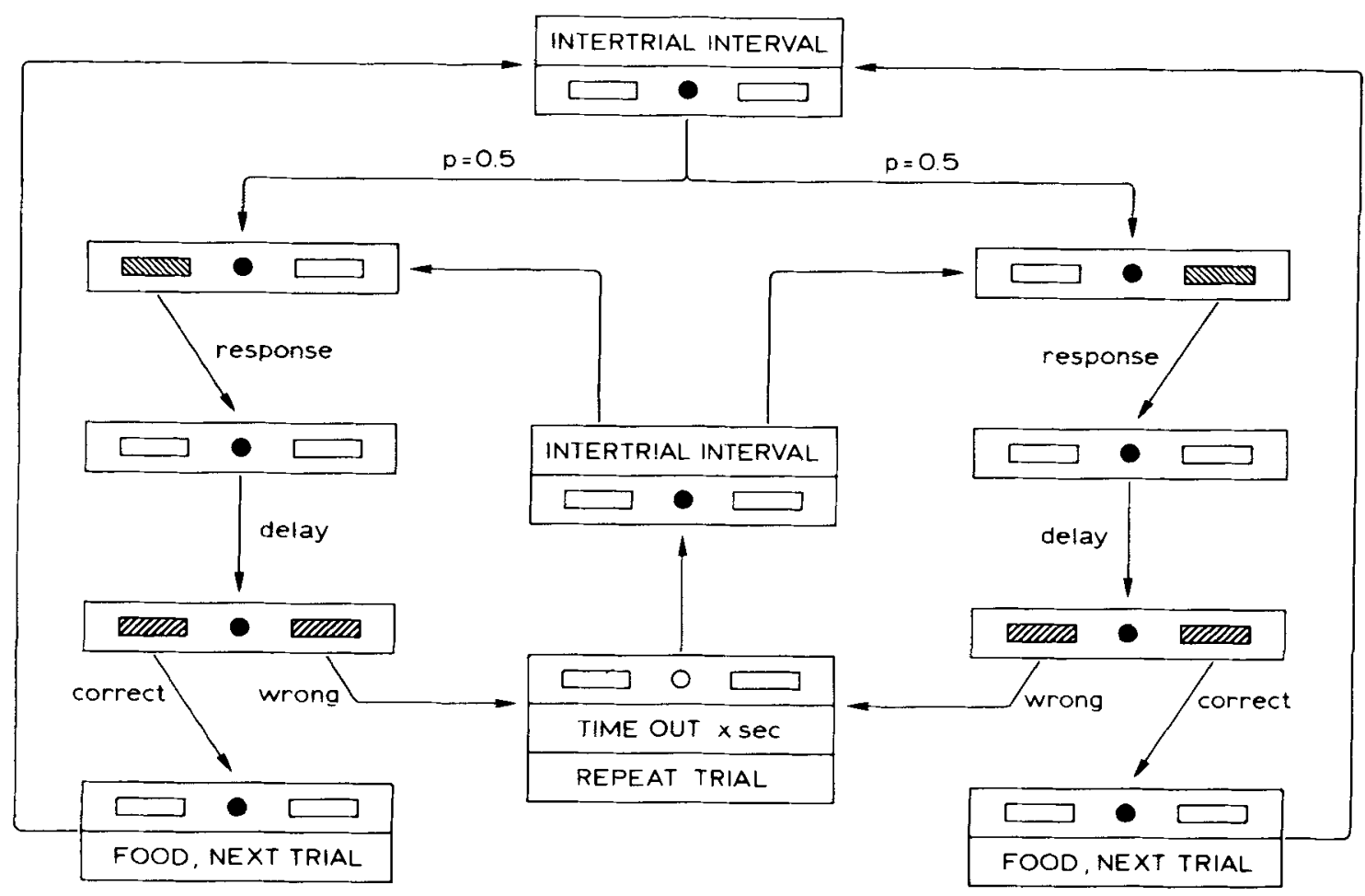

Figure 1. Schematic overview of the operant delayed spatial matching procedure.

responses is shown for the initial presentation of one of the intervals (solid lines), as well as for the trials that were repeated once the initial trial had been incorrectly completed (dotted lines).

The percentages of initial and of repeated correct responses were each analyzed with a four-factor analysis of variance: sex, delay interval $(0,5,10$, or $20 \mathrm{sec})$, con- dition (matching or nonmatching), and sessions (three blocks of 10 , during each condition). All factors apart from sex were considered repeated measures within subjects. The data were arc-sine transformed before analysis to reduce between-subjects variability (Winer, 1971).

The percentage of initial correct matching and nonmatching responses decreased as the delay interval in-

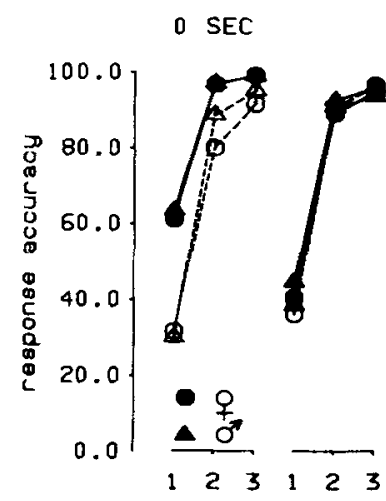

$$
5 \text { SEC }
$$

$10 \mathrm{SEC}$

20 SEC
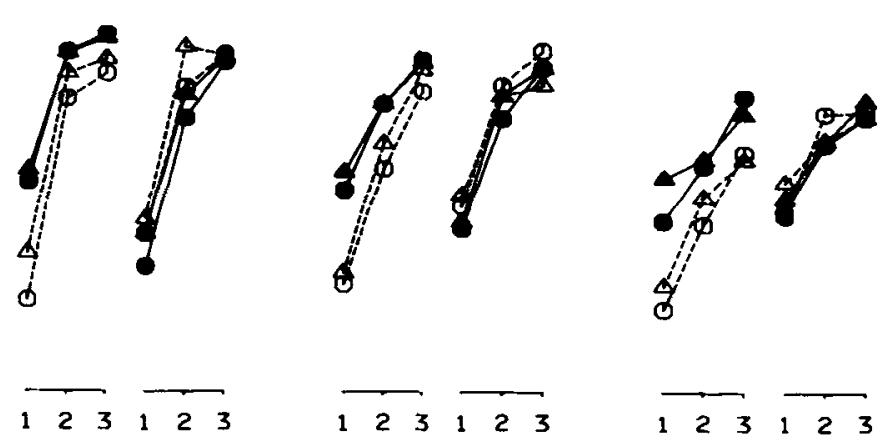

$\begin{array}{llllll}1 & 2 & 3 & 1 & 2 & 3\end{array}$

123123

BLOCKS OF 10 SESSIONS

Figure 2. The percentage correct matching (first three błocks of each subfigure) and nonmatching responses (last three blocks of each subfigure) during initial (solid lines) and repeated (dotted lines) presentations of the different delay intervals for male (triangles) and female (circles) Wistar rats. The percentage of correct responses was calculated to be the number of correct matching (nonmatching) responses divided by the total number of correct plus the total number of incorrect matching (nonmatching) responses. 
Table 1

Total Number of Initial Plus Repeated Trials (Means and Standard Deviations) Required by Males and Females to Reach $80 \%$ Initial Correct Responding During Three Consecutive Sessions for Each Delay Interval

\begin{tabular}{ccccr}
\hline Delay & \multicolumn{2}{c}{ Males } & \multicolumn{2}{c}{ Females } \\
\cline { 2 - 5 } Interval* & $M$ & $S D$ & $M$ & $S D$ \\
\hline \multicolumn{5}{c}{ Matching-to-Position } \\
0 & 211.17 & 33.45 & 177.86 & 50.00 \\
5 & 222.17 & 43.03 & 234.57 & 55.96 \\
10 & 354.83 & 104.41 & 346.14 & 147.98 \\
20 & 598.67 & 132.69 & 561.86 & 97.65 \\
\multicolumn{5}{c}{ Nonmatching-to-Position } \\
0 & 270.83 & 49.69 & 307.14 & 63.13 \\
5 & 365.33 & 131.22 & 379.43 & 73.10 \\
10 & 361.67 & 147.59 & 407.57 & 80.95 \\
20 & 493.00 & 117.17 & 459.86 & 140.83 \\
\hline
\end{tabular}

*In seconds.

creased $[F(3,33)=115.56, p<.001]$. Performance improved as training progressed [sessions, $F(2,22)=$ $345.35, p<.001]$. Differences between males and females were not observed. A significant delay interval $x$ condition interaction $[F(3,33)=53.26, p<.001]$ confirmed the observations that can be made from Figure 2, which suggest that response accuracy after the change in response requirement from matching to nonmatching was lower than that observed before the change, during the short, but not during the longer, delay intervals.

Similar observations were made with respect to the percentage of correct matching and nonmatching responses on repeated (initially incorrect) trials. Response accuracy decreased as the delay interval increased $[F(3,33)=$ $42.88, p<.001]$, whereas performance improved as training progressed $[F(2,22)=271.83, p<.001]$. Again, sex differences were not observed. The significant delay interval $\times$ condition interaction $[F(3,33)=$ $4.84, p<.01$ ] confirmed that during the longer delays $(10$ and $20 \mathrm{sec})$, the percentage of repeated correct responses was higher after the change from matching to nonmatching than it was before the change, but that this was not the case during the shorter delays.

Separate analyses of variance, involving the factors sex, delay interval, and condition, were then conducted on the percentage of initial and repeated correct responses during the 10 sessions before, and the 10 sessions after, the change in experimental conditions. The change in condi- tions caused a decrease in response accuracy during initial trials that was dependent upon the delay interval; response accuracy decreased more during the short than during the long delay intervals $[F(3,33)=79.96$, $p<.001]$. The percentage of correct repeated trials decreased similarly after the change in experimental conditions; the decrease depended upon the delay interval $[F(3,33)=11.34, p<.001]$, and it was again larger for the short than for the long delays $[F(3,33)=22.49$, $p<.001]$. Again, sex differences were not observed.

Table 1 gives an overview of the total number of trials (initial and repeated) required by males and females to reach $80 \%$ initial correct responses during three consecutive sessions for each delay duration. When $80 \%$ correct initial responses were not reached within 30 sessions, the total number of initial and repeated trials during these 30 sessions were calculated and analyzed. Analysis of variance involving the factors sex, delay interval, and condition revealed that the number of trials to reach $80 \%$ initial correct responses increased as the delay interval increased $[F(3,33)=30.57, p<.001]$, and that it was affected by the change in experimental conditions $[F(1,11)$ $=65.22, p<.001]$. Moreover, when a comparison was made between matching and nonmatching efficiency before and after the change in experimental conditions, it increased during the short delays, while decreasing during the longer delays [delays $\times$ condition: $F(3,33)=$ $6.53, p<.001]$.

Overall nose-poke activity (nose pokes/minute) and delay intervals obtained during the last five sessions of each experimental condition are shown in Table 2 . The intervals obtained depended upon the occurrence of nose pokes during the delay. Males responded significantly faster than females during the delays $[F(1,11)=9.63, p<.01]$, but the obtained delay intervals did not differ between the sexes $[F(1,11)=4.18$, n.s. $]$.

\section{DISCUSSION}

The results of the present experiment showed that male and female Wistar rats did not differ in the acquisition of operant delayed spatial matching and nonmatching performance. Males and females were thus equally capable of storing information about spatially defined stimuli during delay intervals that were as long as those in the present experiment. These results confirm observations from other

Table 2

Means and Standard Deviations for Delay Intervals Obtained and Overall Nose-Poke Activity (Nose Pokes/Minute) for Males and Females During the Last Five Sessions of Each Condition

\begin{tabular}{|c|c|c|c|c|c|c|c|c|}
\hline \multirow{3}{*}{$\begin{array}{c}\text { Scheduled } \\
\text { Delay Interval* }\end{array}$} & \multicolumn{4}{|c|}{ Males } & \multicolumn{4}{|c|}{ Females } \\
\hline & \multicolumn{2}{|c|}{ Matching-to-Position } & \multicolumn{2}{|c|}{ Nonmatching-to-Position } & \multicolumn{2}{|c|}{ Matching-to-Position } & \multicolumn{2}{|c|}{ Nonmatching-to-Position } \\
\hline & $M$ & $S D$ & $M$ & $S D$ & $M$ & $S D$ & $M$ & $S D$ \\
\hline 0 & 2.74 & 0.86 & 2.50 & 0.63 & 3.34 & 1.14 & 2.49 & 0.43 \\
\hline 5 & 7.04 & 0.41 & 7.18 & 0.64 & 7.80 & 0.46 & 8.55 & 2.83 \\
\hline 10 & 12.01 & 0.43 & 12.00 & 0.53 & 12.46 & 0.47 & 12.75 & 1.18 \\
\hline 20 & 22.13 & 0.44 & 21.97 & 0.45 & 23.46 & 2.42 & 22.44 & 0.39 \\
\hline Nose pokes/min & 84.70 & 11.37 & 89.28 & 7.54 & 58.09 & 12.81 & 64.00 & 29.40 \\
\hline
\end{tabular}

*In seconds. 
experiments in which behavioral differences between the sexes with respect to memory functions were also not observed (van de Poll et al., 1978; van Haaren, Wouters, \& van de Poll, 1987; van Hest et al., 1988a, 1988b). They thus seem to support the proposition that sex differences in memory functioning may be observed only when other differences between the sexes (e.g., response perseveration, locomotor activity, reactivity to shock) are allowed to interfere with the assessment of memory functioning.

Other experiments have suggested that males are less likely than females to adapt to changes in environmental contingencies. Males have been shown to be less likely than females to reverse a previously well-established freeoperant discrimination performance (Millar, 1975) or to refrain from responding in the presence of a conditional stimulus that previously had been paired with the presentation of response-independent food in autoshaping procedures (van Haaren, van Hest, \& van de Poll, 1987). Differences between males and females were not observed in the present experiment when subjects were required to change from a sample matching to a sample nonmatching response in order for reinforcement to be presented. These results suggest that males and females do not possess basically different abilities in adapting to changes in environmental contingencies. It may be the case that the observed behavioral differences between the sexes in discrimination-reversal performance reflect differences in the acquired associative strength of a conditioned or discriminative stimulus. On the other hand, these discrepancies may also be a function of sex differences in freeoperant response rates, which have frequently been observed in other experiments. For instance, males have been shown to be more likely than females to press a lever that signals the delivery of response-independent food in autoshaping procedures (van Haaren, van Hest, \& van de Poll, 1987; van Hest \& van Haaren, 1989). Males also respond more often when food presentations are responsedependent, both in procedures that selectively reinforce low response rates (Millar, 1975; van Hest et al., 1987a) and in procedures in which high response rates are required for food to be presented (Heinsbroek, van Haaren, Zantvoord, \& van de Poll, 1987; van Haaren, Heinsbroek, Louwerse, \& van de Poll, 1986). Available data suggest that high response rates are more resistant to change than lower response rates (Blackman, 1968; Nevin, 1974), which might explain the less efficient discriminationreversal performance of males in free-operant conditioning procedures. Other experiments, however, will have to be designed to investigate this notion explicitly.

The results of the present experiments also suggest that males and females were more likely to adapt to changes in experimental contingencies during the longer delay intervals. These observations suggest that responses executed in close temporal proximity resist change more than do responses separated by longer intervals. The importance of this observation for the study of the development and, more importantly, the disruption of memory functioning will have to be substantiated in future experiments.
In summary, the results of the present experiment have shown that intact male and female Wistar rats are equally capable of acquiring and performing operant delayed spatial matching and nonmatching responses. The results thus seem to support the notion that the occurrence of behavioral differences between the sexes in simple and complex schedules of reinforcement depends upon specific parameters of the experimental procedures in which they are investigated. It seems reasonable to propose that contradictory evidence obtained in other experimental procedures needs to be similarly evaluated, before accurate predictions with respect to the role of gonadal hormones in learning and memory can be evaluated.

\section{REFERENCES}

BARRETT, R. J., \& RAY, O. S. (1970). Behavior in the open field, Lashley III maze, shuttle-box, and Sidman avoidance as a function of strain, sex, and age. Developmental Psychology, 3, 73-77.

BEATTY, W. W. (1979). Gonadal hormones and sex differences in nonreproductive behaviors in rodents: Organizational and activational influences. Hormones \& Behavior, 12, 112-163.

Blackman, D. E. (1968). Response rate, reinforcement frequency, and conditioned suppression. Joumal of the Experimental Analysis of Behavior, 11, 503-516.

Davenport, J. W., Hagquist, W. W., \& Rankin, G. R. (1970). The symmetrical maze: An automated closed-field test series for rats. $B e-$ havior Research Methods \& Instrumentation, 2, 112-119.

Dawson, J. L. M., Cheung, Y. M., \& LaU, R. T. S. (1975). Developmental effects of neonatal sex hormones on spatial and activity skills in the white rat. Biological Psychology, 3, 213-229.

Heinsbroek, R. P. W., Feenstra, M. G. P., Boon, P., van Haaren, F., \& VAN DE POLL, N. E. (in press). Sex differences in passive avoidance depend on the integrity of the central serotonergic system. Pharmacology, Biochemistry \& Behavior.

Heinsbroek, R. P. W., van HaAren, F., \& van de Poll, N. E. (1988). Sex differences in passive avoidance behavior of rats: Sex-dependent susceptibility to shock-induced behavioral depression. Physiology \& Behavior, 43, 201-206.

Heinsbroek, R. P.W., van HaAren, F., Zantvoord, F., \& Van de Poll, N. E. (1987). Sex differences in response rates during random ratio acquisition: Effects of gonadectomy. Physiology \& Behavior, 39, 269-272.

Hurwitz, H. M. B., \& Davis, H. (1983). Depriving rats of food: A reappraisal of two techniques. Journal of the Experimental Analysis of Behavior, 40, 211-213.

Krasnoff, A., \& Weston, L. M. (1976). Puberal status and sex differences: Activity and maze behavior in rats. Developmental Psychobiology, 9, 261-269.

Millar, R. D. (1975). Free-operant comparisons of wild and domestic Norway rats. Journal of Comparative \& Physiological Psychology, 89, 913-922.

NeVIN, J. A. (1974). Response strength in multiple schedules. Journal of the Experimental Analysis of Behavior, 21, 389-408.

SNAPPER, A. C., \& INGLIS, G. B. (1985). SKED-11: Reference manual. Kalamazoo, MI: State Systems, Inc.

van de Poll, N. E., de Bruin, J. P. C., van Dis, H., \& van Oyen, H. G. (1978). Gonadal hormones and the differentiation of sexual and aggressive behavior and learning in the rat. In $\mathbf{M}$. A. Corner, R. E. Baker, N. E. van de Poll, D. F. Swaab, \& H. B. M. Uylings (Eds.), Maturation of the nervous system: Progress in brain research (Vol. 48, pp. 309-325). Amsterdam: Elsevier.

van HaAren, F., de Bruin, J. P. C., Heinsbroek, R. P. W., \& Van DE PoLL, N. E. (1985). Delayed spatial response alternation: Effects of delay-interval duration and lesions of the medial prefrontal cortex on response accuracy of male and female Wistar rats. Behavioural Brain Research, 18, 41-49. 
van HaAren, F., Heinsbroek. R. P. W., Louwerse, A., \& van de Poll, N. E. (1986). d-Amphetamine differentially affects low, but not high response rates of male and female Wistar rats. Psychopharmacology, 89, 73-76.

Van HaAren, F., \& Van de Poll, N. E. (1984a). The effects of a choice alternative on sex differences in passive avoidance behavior. Physiology \& Behavior, 32, 2i1-216.

Van HaAREN, F., \& VAN DE Poll, N. E. (1984b). The number of preshock trials affects sex differences in passive avoidance behavior. Physiology \& Behavior, 33, 269-272.

van HaAren, F., \& van de Poll, N. E. (1986). Effects of light intensity on passive avoidance behavior of male and female Wistar rats Physiology \& Behavior, 36, 123-125.

van Haaren, F., van Hest, A., \& van de Poll, N. E. (1987). Acquisition and reversal of a discriminated autoshaped response in male and female rats: Effects of long or short and fixed or variable intertrial interval durations. Learning \& Motivation, 18, 220-233.

van Haaren, f., Wouters, M., \& van de Poll, N. E. (1987). Ab sence of behavioral differences between male and female rats in differ ent radial-maze procedures. Physiology \& Behavior, 39, 409-412.

van Hest, A., \& VAN HAaREN, F. (1988). Cooperative behavior in female, but not in male Wistar rats. Manuscript submitted for publication. van Hest, A., \& van HaAREN, F. (1989). The effects of gonadectomy and chronic testosterone suppletion on the autoshaped response of male and female Wistar rats, Bulletin of the Psychonomic Society, 27, 45-48.

van Hest, A., van Haaren, F., \& van de Poll, N. E. (1987a). Be havioral differences between male and female Wistar rats on DRL schedules: Effect of stimuli promoting collateral activities. Physiology \& Behavior, 39, 255-261.

van Hest, A., van Haaren, F., \& van de Poll, N. E. (1987b). Behavioral differences between male and female $W$ istar rats in food rewarded lever holding. Physiology \& Behavior, 39, 263-267.

van Hest, A., van HaAren, F., \& van de Poll, N. E. (1988a). Delayed response alternation: Effects of stimulus presentations during the delay interval on response accuracy of male and female Wistar rats: Bulletin of the Psychonomic Society, 26, 141-144.

van Hest, A., van Kempen, M., van HaARen, F., \& van de Poll, N. E. (1988b). Memory in male and female Wistar rats: Effects of gonadectomy and stimulus presentations during the delay interval. $B e$ havioural Brain Research, 29, 103-110.

WINER, B. J. (1971). Statistical principles in experimental design. New York: McGraw-Hill.

(Manuscript received February 1, 1988; revision accepted for publication July $2,1988$. )

\title{
Announcement
}

\author{
International Congress of Psychology \\ Brussels, Belgium \\ July 19-24, 1992
}

The next International Congress of Psychology will be held in Brussels, July 19-24, 1992, under the auspices of the International Union of Psychological Science (IUPsyS).

Congress brochures may be obtained from Brussels International Conference Centre, Place des Expositions, Place de Belgique, B-1020 Brussels, Belgium (FAX 32247880 23).

Proposals for the Scientific Program, including symposia, may be sent to the U.S. member of IUPsyS, the National Academy of Science. They should be addressed to Pamela Ebert Flattau, USNC/IUPsyS, National Research Council, GH 186, 2101 Constitution Avenue, N.W., Washington, D.C. 20418. 